\title{
Mean-Field and Monte Carlo Study of a Mixed Ferro-Ferrimagnetic Ternary Alloy
}

\author{
J. Dely, A. BobÁk And D. Horváth \\ Department of Theoretical Physics and Astrophysics, Faculty of Science \\ P.J. Šafárik University, Park Angelinum 9, 04001 Košice, Slovak Republic \\ We present a study of the magnetic properties of a mixed ferro-ferrimag- \\ netic ternary alloy of the type $\mathrm{AB}_{p} \mathrm{C}_{1-p}$ on a cubic lattice consisting of three \\ different Ising spins $S_{\mathrm{A}}=\frac{3}{2}, S_{\mathrm{B}}=2$, and $S_{\mathrm{C}}=\frac{5}{2}$. We employ the mean- \\ field approximation and Monte Carlo simulation to find the compensation \\ temperatures of the system for selected values of the parameters in the model \\ Hamiltonian. In particular, the relation between considered mixed ferro- \\ ferrimagnetic model and magnetic properties of the ternary metal Prussian \\ blue analog such as $\left(\mathrm{Fe}_{p}^{\mathrm{II}} \mathrm{Mn}_{1-p}^{\mathrm{II}}\right)_{1.5}\left[\mathrm{Cr}^{\mathrm{III}}(\mathrm{CN})_{6}\right] \cdot n \mathrm{H}_{2} \mathrm{O}$ is discussed.
}

PACS numbers: 05.10.Ln, 75.10.Hk, 75.50.Gg

\section{Introduction}

A new type of molecular-based magnetic materials, which have both ferromagnetic and antiferromagnetic interactions, has basked in the growing scientific interest in recent years. A significant class of these materials, so-called mixed ferro-ferrimagnets, form ternary metal Prussian blue analogs which exhibit many striking properties such as the photoinduced magnetization effect, magnetic pole inversion and occurence of a compensation point [1-3]. The unusual magnetic behaviour of these compounds challenges to their deeper theoretical investigation. However, due to structural complexity of the Prussian blue analogs, the theoretical description of their properties is difficult and up to now has been performed mainly within the mean-field theory (MFT) [4-6] and particularly by using the Monte Carlo (MC) method [7] for a simple two-dimensional model only. Therefore, in the present paper we employ the $\mathrm{MC}$ simulation in order to study the compensation temperatures of a mixed ferro-ferrimagnetic Ising $\mathrm{AB}_{p} \mathrm{C}_{1-p}$ ternary alloy on a cubic lattice. This model, consisting of three different spins $S_{\mathrm{A}}=\frac{3}{2}$, $S_{\mathrm{B}}=2$, and $S_{\mathrm{C}}=\frac{5}{2}$, can simulate some magnetic features of the Prussian blue analog of the type $\left(\mathrm{Fe}_{p}^{\mathrm{II}} \mathrm{Mn}_{1-p}^{\mathrm{II}}\right)_{1.5}\left[\mathrm{Cr}^{\mathrm{III}}(\mathrm{CN})_{6}\right] \cdot n \mathrm{H}_{2} \mathrm{O}[3]$.

\section{General formulation}

The considered $\mathrm{AB}_{p} \mathrm{C}_{1-p}$ ternary alloy, with a lattice size $L$ and periodic boundary conditions, includes two interpenetrating face-centered cubic lattices, 
each one of $L^{3} / 2$ sites. The A ions of one sublattice are alternately connected with the $\mathrm{B}$ or $\mathrm{C}$ ions randomly located on the other sublattice with concentration $p$ or $1-p$, respectively. Therefore, the Hamiltonian of the ternary alloy we adopt has the form

$$
H=-\sum_{(i, j)} S_{i}^{\mathrm{A}}\left[J_{\mathrm{AB}} S_{j}^{\mathrm{B}} \xi_{j}+J_{\mathrm{AC}} S_{j}^{\mathrm{C}}\left(1-\xi_{j}\right)\right],
$$

where $\xi_{j}$ takes the value of unity (zero) if the site $j$ is occupied by B (C) ion. The nearest-neighbour interactions, $J_{\mathrm{AB}}>0$ and $J_{\mathrm{AC}}<0$, correspond to the Prussian blue analog $[3[$. We choose $L=20$ for the simulations. All initial spin states were randomly assigned. Hereafter, configurations were generated by random passing through the lattice and making single-spin flip attemps at each site. The flips are accepted or rejected according to the Glauber algorithm [8]. Data are generated with $10^{4} \mathrm{MC}$ steps per site after discarding the initial $3 \times 10^{3}$ steps per site.

We calculated the sublattice magnetizations per site

$$
m_{\mathrm{A}}=\frac{2}{L^{3}}\left\langle\left|\sum_{i} S_{i}^{\mathrm{A}}\right|\right\rangle, \quad m_{\mathrm{B}}=\frac{2}{L^{3}}\left\langle\left|\sum_{j=1}^{N_{\mathrm{B}}} S_{j}^{\mathrm{B}}\right|\right\rangle, \quad m_{\mathrm{C}}=\frac{2}{L^{3}}\left\langle\left|\sum_{j=1}^{N_{\mathrm{C}}} S_{j}^{\mathrm{C}}\right|\right\rangle,
$$

where $N_{\mathrm{B}}$ denotes the number of B ions $\left(N_{\mathrm{B}}=p L^{3} / 2\right)$, whilst $N_{\mathrm{C}}$ represents the number of $\mathrm{C}$ ions $\left(N_{\mathrm{C}}=(1-p) L^{3} / 2\right)$ on the same sublattice. With respect to the sign of interactions we defined the total magnetization per site as $M=$ $\left(m_{\mathrm{A}}+m_{\mathrm{B}}-m_{\mathrm{C}}\right) / 2$. We note that at the compensation temperature $T_{\text {comp }}$ the total magnetization of the system vanishes below its critical temperature $T_{\mathrm{c}}$. In order to locate the compensation point we also defined an order parameter per spin $O$ in the form

$$
O=\frac{1}{L^{3}}\left\langle\left|\sum_{i, j}\left(S_{i}^{\mathrm{A}}+S_{j}^{\mathrm{B}}+S_{j}^{\mathrm{C}}\right)\right|\right\rangle
$$

which is equivalent to the average of absolute value of the total magnetization. In the limit of infinite lattice the order parameter $O$ approaches zero value at $T_{\text {comp }}$, but in the case of finite lattices the parameter $O$ reaches only a minimum in the compensation point.

Although the results of MC method are rather reliable, it is always constrained by the computer speed. For this reason we also investigated the system described by the Hamiltonian (1) using the MFT. Consequently, the MFT results have been instructive for the time-consuming MC simulations.

\section{Results and discussion}

Now, let us proceed to the discussion of our numerical results. We investigated the magnetic properties of three-dimensional ternary alloy $\mathrm{AB}_{p} \mathrm{C}_{1-p}$, especially the effect of concentration $p$ and interaction ratio $R=\left|J_{\mathrm{AC}}\right| / J_{\mathrm{AB}}$ on the compensation temperature and total magnetization curves. The MFT predicts a multi-compensation behaviour of system for $R \in(0.87,1.43)$. Therefore, we choose 
the representative value of $R=1.0$ for the MC simulation to verify this prediction. On the other hand, we also examined the special case of the ternary alloy with the interaction ratio $R=2.642$ which corresponds to the above-mentioned Prussian blue analog [3].

In Fig. 1a we present the total magnetization $M$ of the ternary alloy with $R=1.0$ as a function of the temperature for several values of concentration $p$. It is clearly seen from this figure that in the region of transition temperature the MFT and $\mathrm{MC}$ results are, as expected, markedly different. However, at low temperatures
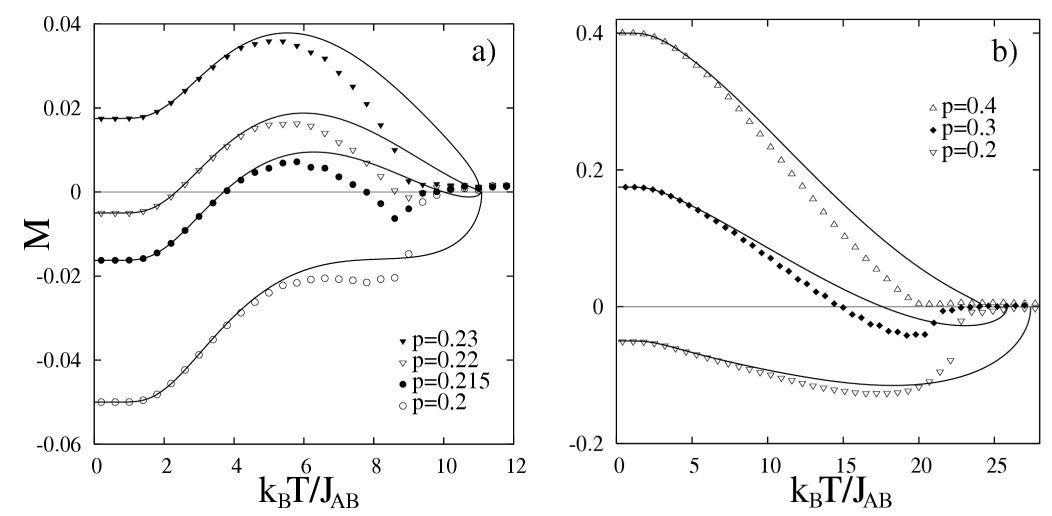

Fig. 1. The temperature dependences of the total magnetization $M$ for ternary alloy $\mathrm{AB}_{p} \mathrm{C}_{1-p}$ with (a) $R=1.0$ and (b) $R=2.642$, for selected values of the concentration $p$. The solid lines and points represent the MFT and MC results, respectively.

we achieved the excellent agreement of both approaches. Further, it is also apparent that the occurrence of the compensation point is very sensitive to the value of $p$. Indeed, the MFT predicts the compensation effect only for $p \in(0.210,0.222)$ while the $\mathrm{MC}$ simulation estimates a little diverging concentration region, namely $p \in(0.213,0.222)$. However, the MFT prediction of two compensation temperatures for the concentration close to $p=0.215$ was confirmed by the MC simulation (see the curve for $p=0.215$ in Fig. 1a). It is worth to remark that the position of low-temperature compensation points acquired within both methods does not differ, but high-temperature compensation point, if there exists, obtained from the MFT is overestimated owing to unreliability of this theory close to the critical region. We note also that the appearance of the compensation point was confirmed by the temperature dependences of the order parameter $O$ for the larger systems ( $L=24$ or 28 ), but due to lack of space they are not presented here.

In the case of $R=2.642$, the magnetization curves of the ternary alloy are plotted in Fig. 1b for several values of $p$. The MFT as well as MC simulation yield one compensation point for the concentration close to $p=0.3$. However, it is known that compound $\left(\mathrm{Fe}_{p}^{\mathrm{II}} \mathrm{Mn}_{1-p}^{\mathrm{II}}\right)_{1.5}\left[\mathrm{Cr}^{\mathrm{III}}(\mathrm{CN})_{6}\right] \cdot n \mathrm{H}_{2} \mathrm{O}$ exhibits the compensation behaviour for $p=0.4$ [3]. As seen from Fig. 1b, neither the MFT nor the MC 
simulation confirm this experimental fact. We believe that this discrepancy with the experiment is coming from the fact that the structure of the studied alloy is mismatched to that of the Prussian blue analog which consists of two sublattices having different numbers of the nearest neighbours. These values for the Prussian blue analog are fixed and controlled by the stoichiometry of the system. Detailed analysis of the effect of different numbers of nearest neighbours of the $\mathrm{A}$ and $\mathrm{X}$ ( $\mathrm{X}=\mathrm{B}$ or $\mathrm{C}$ ) ions on magnetic properties of the ternary alloy is now under way and will be reported elsewhere.

\section{Acknowledgments}

This work was supported by Grants VEGA (Grant no. 1/2009/05) and APVT (Grant no. 20-005204).

\section{References}

[1] K. Hashimoto, S. Ohkoshi, Philos. Trans. R. Soc. Lond. A 357, 2977 (1999).

[2] O. Sato, T. Iyoda, A. Fujishima, K. Hashimoto, Science 272, 704 (1996).

[3] S. Ohkoshi, S. Yorozu, O. Sato, T. Iyoda, A. Fujishima, K. Hashimoto, Appl. Phys. Lett. 70, 1040 (1997).

[4] A. Bobák, F. O. Abubrig, T. Balcerzak, Phys. Rev. B 68, 224405 (2003).

[5] V.V. Kostyuchenko, A.K. Zvezdin, J. Magn. Magn. Mater. 258-259, 548 (2003).

[6] J. Dely, A. Bobák, Physica B 388, 49 (2007).

[7] G.M. Buendía, J.E. Villarroel, J. Magn. Magn. Mater. 310, e495 (2007).

[8] R.J. Glauber, J. Math. Phys. 4, 294 (1963). 